\title{
Polaron-exciton model of resonance energy transfer
}

\author{
T. Markvart \\ School of Engineering Sciences, University of Southampton, Southampton SO17 1BJ, United Kingdom \\ R. Greef \\ School of Chemistry, University of Southampton, Southampton SO17 1BJ, United Kingdom
}

(Received 30 March 2004; accepted 2 July 2004)

\begin{abstract}
It is shown that Förster's expression for the electronic energy transfer rate can be recast in a form predicted for exciton motion that interacts strongly with molecular vibrations. Using a simple model based on the Kennard-Stepanov theory, Förster's expression for the spectral overlap is shown to be of a thermally activated form, as obtained previously by multiphonon theory. In contrast, the high-frequency internal vibrations contribute a factor which results from tunneling through a potential barrier between potential curves in the configuration coordinate diagram. We thus show that resonance energy transfer is equivalent to phonon-assisted hopping of a trapped excitonic polaron. (C) 2004 American Institute of Physics. [DOI: 10.1063/1.1786575]
\end{abstract}

\section{INTRODUCTION}

The transfer of electronic excitation energy (often called resonance energy transfer) continues to attract much research interest. The celebrated Förster-Dexter theory ${ }^{1,2}$ has been used with success to explain a wide range of phenomena, including the energy transport in photosynthesis, ${ }^{3}$ molecular beacons, ${ }^{4}$ as well as applications to modern optoelectronic devices. ${ }^{5}$ Complementing the Förster-Dexter theory, the multiphonon theory of resonance energy transfer ${ }^{6}$ - developed in parallel with the theories of intermolecular electron transfer ${ }^{7}$ - was applied with success to energy transfer in molecular crystals ${ }^{8}$ and photosynthetic light harvesting. ${ }^{9}$ In a simplified two-phonon form, ${ }^{10,11}$ this theory has achieved a particular success, projecting a compromise between the complexity of the full problem and the desire to obtain an analytical solution. Interesting aspects of resonance energy transfer have been revealed by incorporating dye molecules in zeolites. ${ }^{12}$

In recent years, renewed interest in the theoretical foundations of this process has been stimulated by new experimental evidence pertaining, in particular, to the energy transport in photosynthesis. Included in this picture are attempts to unify incoherent Förster transfer and the coherent propagation of wavelike excitons, ${ }^{13-15}$ studies of ultrafast transfer before vibrational equilibrium is attained, ${ }^{16,17}$ and the link between nonradiative transfer and photon exchange. ${ }^{18}$

The principal aim of this paper is to bring together the two seemingly disparate pictures of resonance energy transfer which emerge from an analysis of the spectroscopic analysis and from the microscopic multiphonon theories. We demonstrate, in particular, that the notion of Förster transfer coincides with the phonon-assisted hopping of a trapped excitonic polaron. Using a simple yet quite general model for the molecular spectrum we demonstrate in Sec. II. that the Förster transfer rate can be cast in an activation form, where the activation energy is a simple function of the spectral Stokes' shift of the 0-0 line. We then go on to show in Sec.
III. how this result can be understood by applying the notion of exciton to small molecular polaron. ${ }^{19,20}$ Although the exciton-polaron link has been known for some time ${ }^{21,22}$ it is here reviewed using a consistent semiclassical methodology which produces accurate results at all temperatures. It is shown, in particular, that a low-temperature tunneling approximation for a quantum mode can be obtained by using a simple tunneling formula for a potential barrier which follows from the configuration coordinate (CC) diagram.

For simplicity, we consider only the energy transfer between identical molecules. This makes it possible to illustrate the theory with transparent results and show that the results are sufficiently accurate to be of practical value.

\section{THE FÖRSTER RATE BETWEEN IDENTICAL MOLECULES WITH GAUSSIAN SPECTRAL LINES}

The focus of this paper are molecules with "textbook" type molecular absorption and fluorescence spectra shown in Fig. 1. This picture also assumes that the vibrational parameters in the excited and ground electronic states are identical, as manifested by the mirror symmetry of the spectra. The salient features of the spectra-the vibrational sidebands and line broadening - can be ascribed to two types of modes, with qualitatively different properties. The vibrational sidebands are invariably due to internal modes whose frequency is usually sufficiently high to necessitate description in quantum mechanical terms. In contrast, the broadening of the spectral lines is due to low frequency classical modes of the solvent atoms surrounding the molecules. The theory of line shapes has been developed in general terms ${ }^{23}$ but here we shall strive for the simplicity of the Jortner-Sarai-Yomosa two-phonon model ${ }^{6,9}$ and consider only one mode of each type in the analysis.

The theory can be presented in a particularly clear form by using the CC diagram where each vibrational motion in the electronic ground or excited states can be represented by the harmonic potentials: 


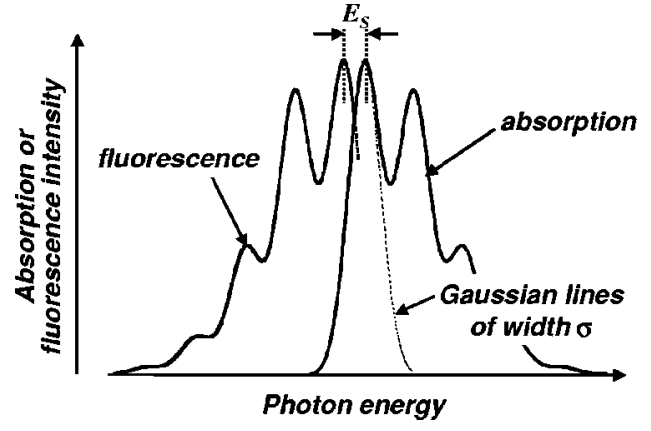

FIG. 1. A schematic diagram of the absorption and fluorescence spectra considered in the present work.

$$
\begin{aligned}
& E_{g}(q)=\frac{1}{2} \omega^{2} q^{2}, \\
& E_{\text {exc }}(q)=E_{0}+\frac{1}{2} \omega^{2}\left(q-q_{0}\right)^{2} .
\end{aligned}
$$

Here, $\omega$ is the vibrational frequency, $q$ is a (normalized) vibrational coordinate, and $q_{0}$ is the relative displacement of the equilibrium positions [Fig. 2(a)].

The displacement $q_{0}$ which characterizes the excitonphonon interaction has different observable interpretation for the quantum and classical modes. At moderate temperatures, the vibrational sidebands are usually well resolved and the intensity of the $0 \rightarrow n$ sideband is proportional to (see, for example, Ref. 23, p. 220)

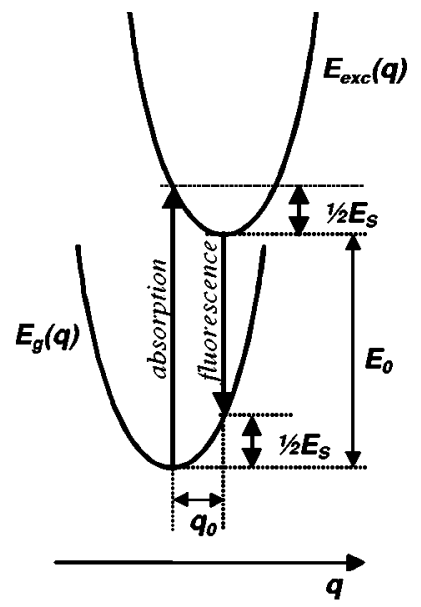

(a)

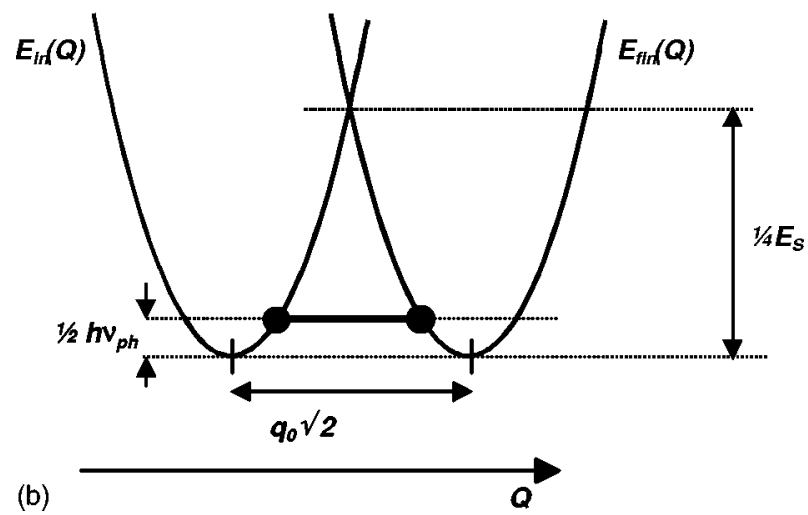

FIG. 2. The configuration coordinate diagrams for the optical processes (a) and for the excitation transfer (b). The dumbbell line in (b) indicates the integration path in the integral (16).

$$
S^{n} e^{-S / n !}
$$

where $S$ is the dimensionless Huang-Rhys factor related to displacement $q_{o}$ by

$$
S=\omega q_{0}^{2} / \hbar .
$$

For the classical low frequency solvent modes, the displacement $q_{o}$ gives the Stokes shift energy $E_{S}$ and the linewidth $\sigma:$

$$
\begin{aligned}
& E_{S}=\omega^{2} q_{0}^{2}, \\
& \sigma=\sqrt{E_{S} k_{B} T} .
\end{aligned}
$$

Equation (4) follows directly from the CC diagram in Fig. 2(a). The expression for the linewidth (5) can be obtained from the general theory of lines shapes in the slow modulation limit (see Ref. 23, p. 222) but can also be derived for Gaussian spectral lines ${ }^{24}$ by using the Kennard-Stepanov relation. ${ }^{25,26}$ A simple model for Eq. (5) can be found in Ref. 22.

We now turn to the Förster transfer. Using Fermi's golden rule, the resonance energy transfer rate can be written as $^{22}$

$$
W=\frac{2 \pi}{\hbar}|\mathrm{V}|^{2} \int \varphi(E) \alpha(E) d E .
$$

Here, $E$ is the energy transferred in the transition, and $\alpha(E)$ and $\varphi(E)$ are the 0-0 absorption and fluorescence line-shape functions of the spectrum in Fig. 1, normalized to unity on the energy scale. In the Gaussian approximation,

$$
\begin{aligned}
& \alpha(E)=\frac{e^{-S}}{\sigma \sqrt{2 \pi}} \exp \left(-\frac{\left(E-E_{o}-\frac{1}{2} E_{S}\right)^{2}}{2 \sigma^{2}}\right), \\
& \varphi(E)=\frac{e^{-S}}{\sigma \sqrt{2 \pi}} \exp \left(-\frac{\left(E-E_{o}+\frac{1}{2} E_{S}\right)^{2}}{2 \sigma^{2}}\right),
\end{aligned}
$$

where $E_{o}$ is the electronic energy of the optical transition. The electronic interaction matrix element $V$ in Eq. (6) can be written in terms of the scalar product of the transition dipole vectors $\mathbf{m}_{1}$ and $\mathbf{m}_{2}$ in the form

$$
\mathrm{V}=\frac{\mathbf{m}_{1} \cdot \mathbf{m}_{2}}{n^{2} R^{3}},
$$

where $R$ is the distance between the molecules and $n$ is the refractive index of the medium.

Substituting Eq. (7) with Eqs. (4) and (5) into Eq. (6) and evaluating the integral then gives

$$
W=\frac{|\mathrm{V}|^{2}}{\hbar} e^{-2 S} \sqrt{\frac{\pi}{E_{S} k_{B} T}} e^{-E_{a} / k_{B} T}
$$

where

$$
E_{a}=E_{S} / 4 \text {. }
$$

Thus, based on a simple but general model of the spectral lines, resonance energy transfer can be described as an activation-type process, with activation energy equal to a quarter of the Stokes' shift. We note that the internal (quantum) and solvent (classical) modes contribute different fac- 
tors to the transition rate. We shall see presently that the difference lies precisely in the different ways that the classical and quantum modes surmount the potential barrier to vibrational motion: by thermal activation or by quantum tunneling.

\section{SMALL..POLARON TREATMENT OF THE FORSTER TRANSFER RATE}

The activation-type law (9), derived here from the molecular spectra, has been obtained previously as the classical limit of the multiphonon theory of nonradiative transition rates. We shall show that this is no coincidence: the two factors in Eq. (9) can be derived from a different viewpoint by considering a new $\mathrm{CC}$ diagram tailored to describe the excitation energy transfer.

To this end, let us consider configuration coordinates formed from linear combinations $Q$ and $Q^{\prime}$ of the vibrational modes pertaining to the donor and acceptor molecules (indicated by subscripts $a$ and $d$ ),

$$
\begin{aligned}
& Q=\frac{1}{\sqrt{2}}\left(q_{a}-q_{d}\right), \\
& Q^{\prime}=\frac{1}{\sqrt{2}}\left(q_{a}+q_{d}\right) .
\end{aligned}
$$

The configuration coordinate diagram contains the energies of the initial and final states before and after the Förster transfer. In terms of the energies of the molecular states, these are

$$
\begin{aligned}
E_{\text {in }}\left(q_{a}, q_{d}\right) & =E_{g}\left(q_{a}\right)+E_{\text {exc }}\left(q_{d}\right) \\
& =\frac{1}{2} \omega^{2} q_{a}^{2}+E_{o}+\frac{1}{2} \omega^{2}\left(q_{d}-q_{o}\right)^{2}, \\
E_{\text {fin }}\left(q_{a}, q_{d}\right) & =E_{g}\left(q_{d}\right)+E_{\text {exc }}\left(q_{a}\right) \\
& =\frac{1}{2} \omega^{2} q_{d}^{2}+E_{o}+\frac{1}{2} \omega^{2}\left(q_{a}-q_{o}\right)^{2},
\end{aligned}
$$

or, in terms of the coordinates $Q$ and $Q^{\prime}$,

$$
\begin{aligned}
& E_{i n}(Q)=\frac{1}{2} \omega^{2}\left(Q+\frac{q_{o}}{\sqrt{2}}\right)^{2}+\varepsilon\left(Q^{\prime}\right), \\
& E_{f i n}(Q)=\frac{1}{2} \omega^{2}\left(Q-\frac{q_{o}}{\sqrt{2}}\right)^{2}+\varepsilon\left(Q^{\prime}\right),
\end{aligned}
$$

where

$$
\varepsilon\left(Q^{\prime}\right)=E_{0}+\frac{1}{2} \omega^{2}\left(Q^{\prime}-\frac{q_{o}}{\sqrt{2}}\right)^{2} .
$$

The principal feature to note is that the $\mathrm{CC}$ diagram described by Eq. (14) is effectively independent of $Q^{\prime}$. The coordinate $Q^{\prime}$ enters only through the function $\varepsilon$ which is common to both energies $E_{\text {in }}$ and $E_{\text {fin }}$. The energy $\varepsilon\left(Q^{\prime}\right)$ therefore acts as a form of reference point from which these energies are reckoned but it remains unchanged during the transition. The $\mathrm{CC}$ diagram which describes the excitation transfer therefore contains only the configuration coordinate $Q$ [Fig. 2(b)].

Just like the optical transitions, the nonradiative excitation transfer must comply with the Franck-Condon principle which states that $Q$ cannot change during the transition. Since there is no external energy source, energy must be conserved, and the transition can only occur at the crossing point $Q=0$ of the potential curves (14). In terms of the molecular coordinates this condition becomes $q_{a}=q_{d}$; in addition, each $q_{a}$ and $q_{d}$ must also remain constant in the transition.

The curves (14) thus form a potential barrier of height $\omega^{2} q_{0}^{2} / 4$ and width $Q_{0}=q_{0} \sqrt{2}$ at the base. In the classical mode, this motion takes place by thermal activation over the barrier; in the quantum mode, it occurs by tunneling. It should be noted that the Huang-Rhys factor for exciton transfer is $\omega Q_{0}^{2} / \hbar=2 \omega q_{0}^{2} / \hbar=2 S$; in other words, it is equal to twice the value (4) which is observed from the absorption or fluorescence spectrum of one molecule. This explains the apparent paradox which has been used to reject the application of the semiclassical approximation to low-temperature energy transfer. Our treatment above does not consider the

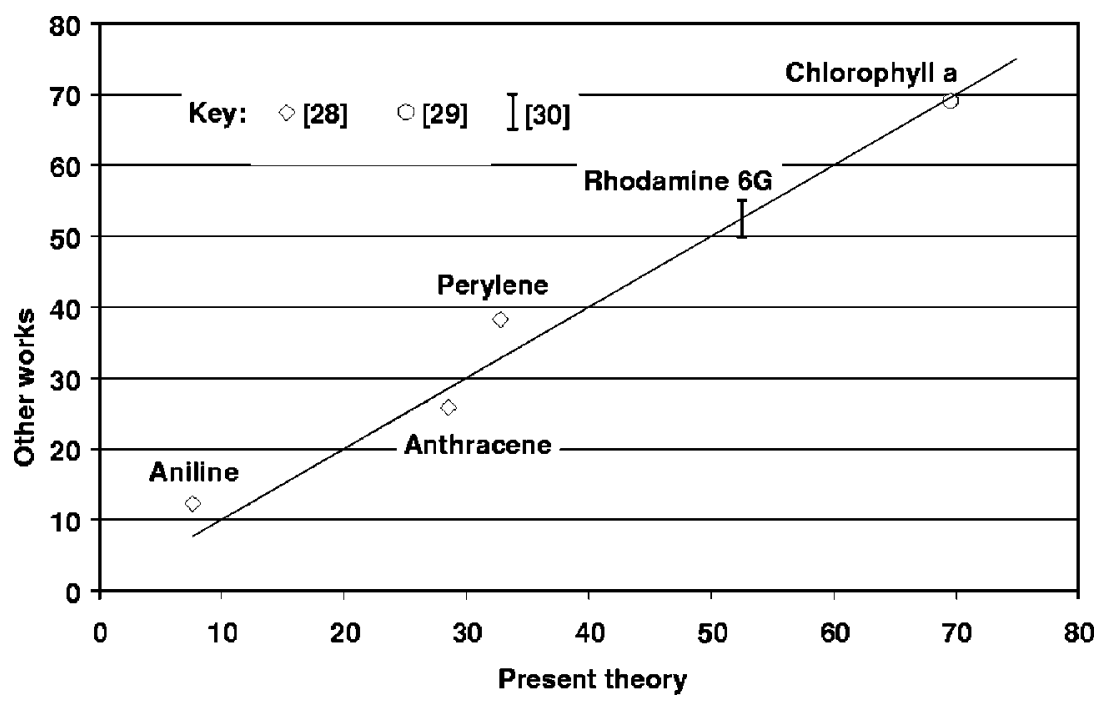

FIG. 3. The comparison of the Förster distance $R_{o}$ (in angstroms) calculated using Eq. (9) with the results obtained by other workers (shown by points). The line shows a locus of points predicted by Eq. (9). The spectral data used as input to Eq. (9) were obtained from Ref. 31; the electronic matrix element was calculated from the natural fluorescence lifetime $\tau_{f}$ using the expression for the transition dipole moment $m^{2}=3 h^{4} c^{3} / 64 \pi^{4} n E_{o}^{3} \tau_{f}$. 
question of coupled modes and assume that the vibrations associated with each molecule are independent; a detailed discussion of these aspects can be found in Ref. 8 .

The thermally activated case has been discussed in a number of papers ${ }^{19,21}$ where different equivalent derivations can be found. A consistent discussion of the role played by the quantum mode of internal vibrations in semiclassical terms, however, is less well documented. Let us consider the transmission coefficient for tunneling through potential barrier in the CC diagram as given by the WKB theory,

$$
T=\exp \left\{-\frac{2 \sqrt{2}}{\hbar} \int \sqrt{E(Q)-\frac{1}{2} h \nu_{p h}} d Q\right\}
$$

where $E(Q)$ is the barrier function represented by the functions $E_{i n}$ (for $Q<0$ ) and $E_{f i n}$ (for $Q>0$ ), $\nu_{p h}$ is the vibrational frequency, and the integration is over the range shown in Fig. 2(b). The factor in front of the integral in Eq. (16) reflects the fact that, in the potential functions (14), the mass factor has been set equal to unity. In the semiclassical limit where the expression (16) is applicable, the barrier is much higher than the phonon energy $h \nu_{p h}$. We can therefore neglect the latter and extend the integral to run between the equilibrium minima to obtain

$$
\begin{aligned}
T= & \exp \left\{-\frac{2 \sqrt{2}}{\hbar} \int \sqrt{E(Q)} d Q\right\} \\
= & \exp \left\{-\frac{2 \sqrt{2}}{\hbar}\left[\int_{-q_{0} / \sqrt{2}}^{0} \sqrt{\frac{1}{2} \omega^{2}\left(Q+q_{o} / \sqrt{2}\right)^{2}} d Q\right.\right. \\
& \left.\left.+\int_{0}^{+q_{0} / \sqrt{2}} \sqrt{\frac{1}{2} \omega^{2}\left(Q-q_{o} / \sqrt{2}\right)^{2}} d Q\right]\right\} \\
= & \exp (-2 S),
\end{aligned}
$$

where $S$ is the Huang-Rhys factor (3). The result (17) shows that the Franck-Condon vibrational factor is equal, within the accuracy of the semiclassical approximation, to the transmission coefficient for tunneling through the potential barrier for the exciton motion, thus highlighting the two different interpretations of the factor $e^{-2 S}$.

\section{DISCUSSION AND CONCLUSIONS}

We have shown, by considering resonance energy transfer between identical molecules, that the concept of Förster energy transfer is identical in detail to the transport of a self-trapped excitonic polaron. This transport is thermally activated when assisted by low-frequency solvent phonons, the same vibrations that give rise to the broadening of the spectral lines. Internal vibrations that appear as sidebands in the molecular spectra form a barrier in the CC diagram which is surmounted by quantum-mechanical tunneling. The formulas presented in this work are accurate enough for practical calculations, as shown in Fig. 3 which compares the Förster distance $R_{0}$ calculated using expression (9) with results obtained in the literature for several frequently encountered molecules. In addition to providing an insight into the fundamentals of the process, the theory also provides simple answers to such questions as the temperature dependence of the transfer rate and gives simple recipes to estimate the energy transfer rate in circumstances when the knowledge of the relevant spectral parameters is less than complete.

This picture can be extended to energy transfer between different molecules where the computational complexity, however, conceals the simple physical interpretation of this result. Further parallels can be drawn between the coherent exciton motion and the band propagation of the small polaron which was discussed clearly by Holstein and Friedman in terms of the so-called diagonal and nondiagonal transitions. At larger values of the interaction matrix element (8), the small polaron picture considered in this paper no longer holds and should be replaced by the large polaron theory. A suitable formalism is discussed, for example, in Ref. 27.

\section{ACKNOWLEDGMENTS}

Thanks are due to our colleagues John Dyke, Jeremy Frey, and Peter Landsberg for many useful discussions on issues considered in this paper.

${ }^{1}$ T. Förster, Ann. Phys. 2, 55 (1948).

${ }^{2}$ D. L. Dexter, J. Chem. Phys. 21, 836 (1953).

${ }^{3}$ See, for example, the reviews R. S. Knox, in Excitation Energy Transfer and Migration: Theoretical Considerations, Bioenergetics of Photosynthesis, edited by Govindjee (Academic, New York, 1975), p. 183; R. van Grondelle, J. P. Dekker, T. Gillbro, and V. Sundstrom, Biochim. Biophys. Acta 1187, 1 (1994).

${ }^{4}$ An extensive review of the use of RET in molecular studies can be found in B. W. van der Meer, G. Coker, and S.-Y. Chen, Resonance Energy Transfer: Theory and Data (Wiley, New York, 1994).

${ }^{5}$ See, for example, M. Berggren, A. Dodabalapur, R. E. Slusher, and Z. Bao, Nature (London) 389, 466 (1997).

${ }^{6}$ J. Jortner, J. Chem. Phys. 64, 4860 (1976).

${ }^{7}$ R. A. Marcus, Annu. Rev. Phys. Chem. 15, 155 (1964); see also R. R. Dogonadze, A. M. Kuznetsov, and A. A. Chernenko, Russ. Chem. Rev. 34, 759 (1965).

${ }^{8}$ T. F. Soules and C. B. Duke, Phys. Rev. B 3, 262 (1971).

${ }^{9}$ For a recent review of light harvesting see, for example, V. Sundstrom, T. Pullerits, and R. van Grondelle, J. Phys. Chem. B 103, 2327 (1999).

${ }^{10}$ S. Yomosa, J. Phys. Soc. Jpn. 45, 967 (1978); A. Sarai, Chem. Phys. Lett. 63, 360 (1979).

${ }^{11}$ P. A. Lyle and W. S. Struve, J. Phys. Chem. 95, 4152 (1991).

${ }^{12}$ G. Calzaferri, M. Pauchard, H. Maas, S. Huber, A. Khatyr, and T. Schaafsma, J. Mater. Chem. 12, 1 (2002).

${ }^{13}$ M. Chachisvilis, O. Kuhn, T. Pullerits, and V. Sundström, J. Phys. Chem. B 101, 7275 (1997).

${ }^{14}$ S. K. Sekatskii, Phys. Lett. A 317, 1 (2003).

${ }^{15}$ G. D. Scholes, Annu. Rev. Phys. Chem. 54, 57 (2003).

${ }^{16}$ P. D. Laible, R. S. Knox, and T. G. Owen, J. Phys. Chem. B 102, 1641 (1998).

${ }^{17}$ S. Jang, Y. J. Jung, and R. J. Silbey, Chem. Phys. 275, 319 (2002).

${ }^{18}$ G. J. Daniels, R. D. Jenkins, D. S. Bradshaw, and D. L. Andrews, J. Chem. Phys. 119, 2264 (2003).

${ }^{19}$ T. Holstein, Ann. Phys. 8, 325, 343 (1959).

${ }^{20}$ L. Friedman, Phys. Rev. A135, 233 (1964).

${ }^{21}$ T. Markvart, J. Theor. Biol. 91, 72 (1978).

${ }^{22}$ T. Markvart, Prog. Quantum Electron. 24, 107 (2000).

${ }^{23}$ S. Mukamel, Principles of Nonlinear Optical Spectroscopy (Oxford University Press, New York, 1995).

${ }^{24}$ L. P. Kazachenko, Opt. Spectrosc. 18, 397 (1965).

${ }^{25}$ E. H. Kennard, Phys. Rev. 11, 29 (1918); 28, 672 (1926).

${ }^{26}$ B. I. Stepanov, Dokl. Akad. Nauk BSSR 112, 839 (1957); Izv. Akad. Nauk SSSR 22, 1034 (1958).

${ }^{27}$ T. Meier, Y. Zhao, V. Chernyak, and S. Mukamel, J. Chem. Phys. 107, 3876 (1997). 
${ }^{28}$ I. B. Berlman, Energy Transfer Parameters of Aromatic Compounds (Academic, London, 1973)

${ }^{29}$ L. N. M. Duysens, Prog. Biophys. Mol. Biol. 14, 1 (1964).

${ }^{30}$ G. Jones, MPhil thesis, University of Southampton, 2003; F. L. Arberloa,
P. R. Ojeda, and I. L. Arbeloa, J. Chem. Soc., Faraday Trans. 2, 84 (1988); C. Bojarski and G. Obermueller, Acta Phys. Pol. A 50, 389 (1976).

${ }^{31}$ I. B. Berlman, Handbook of Fluorescence Spectra of Aromatic Molecules (Academic, London, 1971). 